\title{
COVID-19 Management in India: Medical Aspects, Current Concepts, and Evolving Directions
}

\author{
Daya K. Hazra $\quad$ Padmamalika K. Neehazra ${ }^{1} \quad$ Suratwant Hazra ${ }^{1} \quad$ Adarsh N. Segal ${ }^{1}$ \\ ${ }^{1}$ Department of Medicine, Boston Medical Centre, Agra, \\ Uttar Pradesh, India

\begin{abstract}
Address for correspondence Daya K. Hazra, MD, FAMS, PhD, MSc, Boston Medical Centre, Swamibagh, Agra, Uttar Pradesh 282005, India
\end{abstract} \\ (e-mail:Dr_Hazra@yahoo.com).
}

\begin{abstract}
This review deals with the emerging coronavirus disease 2019 (COVID-19) epidemic summarizing current and evolving medical management in India, and also indicating future directions in the light of possible resurgence of this disease as pandemic as well as prospective biological threats resulting from acts of bioterrorism. Clinical accounts both in published or prepublication reports from the frontline epicenters were used, incorporating the evolving flux in guidelines and current controversies, expressed not only in Print media but also video-webinars and conferences constantly in progress. Rather than presenting hard conclusions from the few randomized controlled trials, less formal clinical impressions have been incorporated in the review as it is felt that at this stage of the COVID-19 battle, such data are relevant! Periodic rewriting on this subject is in our

Keywords

- COVID-19

- cytokine storm

- hypoxia

- cardiovascular

- respiratory

- hypercoagulopathy opinion, therefore, eminently desirable (Box format used to allow this). Apart from the respiratory crisis aggravated by cytokine storms, reference is made to the cardiovascular, neurological, renal, gastrointestinal, and cutaneous features, these generalized manifestations being related to the widespread distribution of angiotensin-converting enzyme-2 receptors in the body as well as to the acute inflammatory hypercoagulopathy and disseminated intravascular coagulation typified by hyperacute levels of acute phase reactants as well as the effects of hypoxia-overt or silent!
\end{abstract}

\section{Introduction}

Any account of the medical management of the coronavirus disease 2019 (COVID-19) illness (henceforth called COVID) caused by the severe acute respiratory syndrome coronavirus-2 (SARS-CoV-2) pandemic must be tempered by the disclaimer that this is a rapidly evolving scenario in the context of the spreading pandemic, especially in view of the new knowledge from not only the initial China epicenter, but also from the United States and from Europe. Adding to this are a varying admixture of documented facts, gathering knowledge, and speculations, as well the latest version of ICMR/WHO/CDC/FDA guidelines, which themselves are evolving.

The outlined advice on medical management of COVID is in two categories:

DOI https://doi.org/ 10.1055/s-0040-1718501 ISSN 0379-038X.
1. Logical time-honored management of the acute respiratory distress syndrome (ARDS) resulting from the acute SARS-CoV-2 infection and of the cardiac, neurological, renal, and endocrine aspects.

2. Speculations about the possible benefit from touted interventions. With regard to the latter, our attitude has been that at this stage of the pandemic, it is pedantic to wait for randomized controlled trials to prove or disprove the efficacy of such interventions.

One must also confess that in the absence of limited personal experience in India, the lessons from Italy, the United States, and China as recounted not only in published articles but also videos, talks, webinars, and the like are considered and incorporated. We would particularly commend the $\mathrm{Vu}$ Medi series of videos which give practical visual accounts of

(c) 2020. National Academy of Medical Sciences (India).

This is an open access article published by Thieme under the terms of the Creative Commons Attribution-NonDerivative-NonCommercial-License, permitting copying and reproduction so long as the original work is given appropriate credit. Contents may not be used for commercial purposes, or adapted, remixed, transformed or built upon. (https://creativecommons.org/licenses/by-nc-nd/4.0/).

Thieme Medical and Scientific Publishers Pvt. Ltd. A-12, 2nd Floor, Sector 2, Noida-201301 UP, India 
aspects such as doffing and discarding personal protective equipment (PPE), setting up ventilators, management in the intensive care unit (ICU) during respiratory distress, non-ICU management of mild cases, and providing radiological investigations, etc. ${ }^{1-30}$ We would also emphasize the role of telemedicine in managing non COVID disease while our hospitals are coping with both COVID and non-COVID serious patients.

Further, as we battle this unprecedented threat, one strongly feels that India's preparation for future challenges is a must, some of the salient features of which are listed in - Table 1 (But compare - Supplementary Appendix A, available in the online version). Some of the points mentioned in that preparation plan may sound impossibly expensive or Utopian, but may we submit that wise expenditure ahead of a challenge saves a far greater expenditure/economic/human cost and loss in the future. Bill Gates in the aftermath of the Ebola disaster in 2015 had outlined a plan which might have been of immeasurable value in dealing with today's COVID pandemic! It is heartening to note that the proposed optical fiber high speed internet penetration of our remote villages and the National Digital Health cards as well as the "Make in India" strategy are steps in this direction.

Since many aspects of COVID management are rapidly evolving and are in a state of flux, rather than following the conventional didactic narrative, Boxes have been created summarizing current management/considerations. This would facilitate rapid revision as required with the availability of the new evidence-based information 1, 3, 6, and 12 months in the future.

The COVID picture was initially that of a respiratory syndrome, analogous to the Flu but over the last few months our understanding of the respiratory features has been transformed by the recognition of an insidious silent hypoxia (the walking dead) as well as of both $\mathrm{H}$ and $\mathrm{L}$ forms ( $\boldsymbol{- B o x} \mathbf{A}$ ) warranting different management, capable of differentiation by chest X-ray plain films even without computed tomography (CT) chest scans. ${ }^{31}$

The importance of noninvasive differentiation of cardiological features from atherosclerotic ischemic syndromes has been described in - Box B. The subject of using renin-angiotensin-aldosterone system (RAAS) inhibitors-ACE (angiotensin-converting enzyme) inhibitors or angiotensin receptor blockers in COVID pandemic era is controversial and is discussed in $\boldsymbol{- B}$ Box $\mathbf{C}$. The management of diabetes in COVID patients who are in danger of a cytokine storm, especially the $H$ variety with waterlogged lungs is described in - Box $\mathbf{D}$.

The changing clinical pictures especially the multisystem involvement including gastrointestinal, neurological, renal, endocrine, and dermatological presentations are discussed in - Box E.

- Box $\mathbf{F}$ describes the management of the coagulopathy which must be cautious, so as to avoid precipitating bleeding.

- Box G lists the acute phase reactants that are used to not only arouse suspicion of COVID-19 illness but also assess its severity.

- Box $\mathbf{H}$ outlines the management of the cytokine storm.

- Box I lists factors that adversely affect morbidity and mortality in COVID-19 patients.
Table 1 Post-COVID preparations for the future challenges

Outlining the medical management of COVID cases must be supplemented by some thoughts on India's preparation for future emergencies akin to COVID.

1. Development of the biotechnological knowledge base, including National Therapeutic Biomolecule Centers with associated ICMR supervised low cost limited number clinical trial centers: such centers were proposed by one of the authors (D.K.H.) jointly with Professor V.M. Katoch (then Director, JALMA, and later Director General, Indian Council of Medical Research, and Secretary, Department of Health, MoHFW, Govt. of India) to the Department of Science and Technology, Govt. of India, as far back as 2005.52 This was modeled on the UK Therapeutic Antibody Centre at Cambridge/Oxford. Currently the UK Centre, is part of the Jenner Institute, which is working on vaccines including COVID vaccine.

2. Planning and enforcement of housing norms so that high population density urban slums like Dharavi are not created, and relocation of existing slum dwellers.

3. Manifold strengthening of the medical infrastructure including creation of a National Health Service coverage of the entire population.

4. Capability to manufacture (and later to export) intensive care-oriented medical equipment including ventilators, CPAP, ECMO (extracorporeal membrane oxygenators), extracorporeal endotoxin/cytokine removal cartridges, oxygen concentrators, high flow oxygen delivery devices, pulse oximeters, dialysis devices as well as enhanced capability to make rapid diagnostic kits, active pharmaceutical ingredients (APIs), and biosimilars.

5. Developing capability for rapid clinical trials to validate drugs/strategies and kits, and corresponding pharmacovigilance.

6. Creation of $100 \%$ adult literacy using electronic media so that instructions can be implemented intelligently and expeditiously.

7. Free and compulsory education for all children to at least class 8 level, Pari passu with the above.

8. Minimal food allowance for every family, including two children so that the nutritional health and immune competence of the population is built-up.

9. Salutary punishments for fake drugs.

10. Quality control, with informational technology support to monitor progress toward objectives 1-9.

Abbreviations: COVID, coronavirus disease; CPAP, continuous positive airway pressure; ICMR, Indian Council of Medical Research.

\section{Convalescent Serum/Plasma Therapy and Serologic Testing}

Both for diagnosis as well as for selection of convalescent serum for therapy there is great interest in testing for antibodies.

Antigens are detected by examining the nasopharyngeal swabs. There are in addition tests for the viral nucleic 
Box A Respiratory symptomatology of COVID-19

- To begin with COVID-19 was regarded as a severe flu-like Illness.

- Consisting of predominantly respiratory symptoms.

- Initially, the COVID infection starts with a common cold or flu-like picture: dry cough, sneezes, and nasal/throat congestion. This may progress to breathlessness, and finally to a full-fledged ARDS scenario, sometimes necessitating ventilator support.

- Later, New York intensivists called attention to: Silent Hypoxia p02 well below 85 but patient not complaining about breathlessness (Walking Dead phenomenon!). In this variety, proning posture/intranasal oxygen/corticosteroids/C Pap, and treatment of an impending cytokine storm suspected by high IL-6 levels and acute phase reactants: troponin-T, ferritin, C-reactive protein, and high D-dimers, may avoid the need to intubate and ventilate!

- Radiologically, now $\mathrm{H}$ and $\mathrm{L}$ varieties described on the basis of $\mathrm{CT}$ scans as well as clinical picture: $\mathrm{H}$ phenotype with heavy water-logged lungs, and $\mathrm{L}$ phenotype with lighter, less water-logged lungs. $\mathrm{H}$ types required ventilation, while $\mathrm{L}$ types are harmed by this intervention.

- "It is commonly thought that COVID-19 presents as other coronavirus diseases, with ARDS caused by pneumonia-like thickening of the lungs. However, recent observations suggest that half of all patients present differently, with near-normal breathing mechanics, clearer lungs, and hypoxia caused by poor oxygen transfer to the capillaries. The ARDS-like $\mathrm{H}$ phenotype is characterized by high lung elastance, high lung weight, and high recruitment (potential to re-expand collapsed lung tissue, e.g., closed alveoli); these patients required mechanical ventilation to survive. The corresponding parameters for the L phenotype are low; applying high-pressure ventilation in these cases can injure lungs and precipitate a rapid worsening to ARDS. It is vital to classify patients appropriately. To date, the only way to confirm the phenotypes is through high-resolution computed tomography. This is inhibitory for rapid triage and impossible for hospitals with limited resources. Chest X ray PA achieved" this. ...(Islam and Fleischer, Princeton, May 3, 2020). ${ }^{31}$

Abbreviations: ARDS, acute respiratory distress syndrome; COVID-19, coronavirus disease 2019; IL-6, interleukin-6.

acids. Some of the tests are based on caspase technology, and these are expected to be both rapid and inexpensive, but the problem is to ensure that the particular test can differentiate between intact ribonucleic acid (RNA) and RNA fragments. The usual tests for RNA are based on polymerase chain reactions (PCRs) which require warming as well as involve a turn-around time. Room temperature nucleic acid detection methods are being devised which not only are rapid-detection through a color reaction, but capable of being performed by the subject without going to a laboratory or hospital. If these tests could be performed on saliva, they may conceivably be the basis for a self-monitoring strategy to allow a post-lockout safe resumption of work and the economy. This, of course would depend on
Box B Ischemic cardiological symptomatology of COVID-19

The ischemic cardiac symptoms are important because like the pulmonary features they can contribute to dyspnea. Their differentiation from atherosclerotic ischemic cardiac events is important, preferably noninvasively. Guidance from the cardiac professional bodies on the management of suspected ischemic events in the COVID era, and also with regard to the management of cardiac pathology due to COVID-19 is important to understand.

The cardiological features of COVID-19 include:

- Heart failure/cardiac dilatation/dyskinesia

- BP rise

- Arrhythmias

- Conduction blocks

- Troponin-T elevations even to 5,000

- D-dimer rises even to 30,000

- Angina with or without ST,T changes/Q wave

- May need fibrinolysis clot busting

- Surgical intervention should be avoided to prevent SARSCoV-2 transmission

Abbreviations: BP, blood pressure; COVID-19, coronavirus disease 2019.

individuals willing to do these tests. Simplicity is comparable to that of pregnancy testing in the urine!

There are many kits under evaluation, some of them using CRISP R/Caspase technology to accelerate results.

- Box J describes the current status of antibody testing as per a recent CDC (Centers for Disease Control and Prevention) guidance note..$^{32} \mathrm{~A}$ recent update has also been appended on rapid antigen testing. CDC emphasizes that COVID diagnosis cannot be excluded on the basis of antibody tests.

- Box K summarizes prehospital management of suspected COVID-19 patient and COVID-19 patient contacts.

\section{Controversial Agents Used in COVID-19 Therapy Hydroxychloroquine}

Whether HCQ or chloroquine should be used in COVID-19 patients with cytokine storm,/all patients/contacts/ health care personnel have excited much debate particularly in view of the alleged cardiac complications! QTc prolongation, arrhythmias, and even Torsade-de-pointes and sudden death are alleged to have been induced by these agents. WHO, $2020^{33}$ suspended this arm of the Solidarity trial.

From the point of eye toxicity, HCQ has been thought safer than chloroquine because it does not enter the retinal circulation. It is in use in rheumatoid illness for over 2 decades, and for type 2 diabetes mellitus (T2DM) for the past 4 years. Inter alia, it alters the intracellular $\mathrm{pH}$ and its benefits in diabetes include reduced hyperglycemia, and anti-inflammatory action.

Logically, a careful analysis of COVID-19 disease experience in patients already on such drugs for rheumatoid arthritis/systemic lupus erythematosus/T2DM would be of value and has been suggested by Hazra. ${ }^{34}$ The clinical experience of clinicians at Agra involved in caring for these diseases in pre-COVID era has not been adverse! (Agra Diabetes Forum video meeting, May 28, 2020: Unpublished Discussion). 
Box C RAAS inhibitors and DPP IV inhibitors in the COVID scenario
Experts from Australia, etc. were apprehensive that hypertensives are not predisposed to infections, so why do so many get COVID infection? Was it due to their use of ACE-inhibitor drugs?
Lots of opinions followed!
The virus spike protein enters various organs (respira- tory, cardiac, endothelial, hepatic, pancreatic, etc.,) cells through ACE-2 receptors. How RAAS agents modulate this entry is still under study and is a subject of debate. ACE-inhibitors decrease ACE- 1 receptors.
Angiotensin II receptor I blocked by ARB-the Sartans. Sartans increase ACE- 1 receptors (decrease ACE-2 receptors in experimental animals: controversial in humans).
ACE inhibitors increase bradykinin and may induce cough.
Sacubitril, like ACE inhibitors also increases bradykinin cough and also increases angiotensin II.
Cardiological expert bodies advise that: do not withdraw RAAS agents in heart failure/chronic kidney disease/ diabetics with kidney disease in COVID patients.
But if the indication for RAAS agents is hypertension, the physician can substitute Ca-blockers/diuretics!
But avoid starting these in COVID-19 era!: "Primum no cere": Hippocrates
ACE-2 and DPP-IV enzymes are coronavirus receptors. DPP-IV enzyme is a MERS-CoV receptor, but not a SARS- CoV-2 receptor, that is, not a COVID-19 receptor. ACE-2 and DPP-IV enzymes control inflammation and cardiometabolic physiology.
DPP-IV inhibitors do not meaningfully modify immune response in humans.
COVID-19 hospitalizations are more common among people with diabetes and obesity.
Many diabetics, hypertensives, and heart patients are already on ACE inhibitors/angiotensin receptor blockers. ACE inhibitors block ACE-1 and not ACE-2 enzyme. There is a suggestion that angiotensin receptor block- ers (ARBs) can theoretically reduce coronavirus entry through the ACE-2 receptors but currently, no change in existing RAAS medication is advised by cardiological opinion makers, and some series of studies indicate slightly better outcomes in relation to lung damage if these are continued.
But conclusive data are still awaited. However, fresh initiation of such agents in the COVID-19 patient is best avoided. Of these, ARBs are preferred because ACE inhibitors often cause cough, which can be confusing in COVID-19 situation.

Abbreviations: ACE, angiotensin-converting enzyme; BP, blood pressure; COVID-19, coronavirus disease 2019; DPP-IV, dipeptidyl peptidase-4; MERS-CoV, Middle East Respiratory Syndrome Coronavirus; RAAS, renin-angiotensin-aldosterone system.

However, the measurement of QTc intervals prior to the use of these drugs (to identify green/orange or red categories analogous to traffic signals) has been suggested by United States as well as Indian cardiologists and diabetologists!

To avoid relatively hazardous electrocardiography (EKG) recording in COVID in ICU patients, measuring the QTc from the ICU monitor both prior to and subsequent to HCQ administration has been suggested.

Particular care is needed in patients on azithromycin which itself causes QTc prolongation.
Box D Management of diabetes mellitus in COVID-19 patients

- Old age, diabetes mellitus (DM), heart disease, and even more so, obesity adversely affects complications and mortality amongst COVID-19 sufferers. It is uncertain whether DM increases the attack rate for COVID-19, but it is very clear that the morbidity and mortality is much worse amongst diabetics.

- Acute COVID-19 illness requires reevaluation of any medications used for type $2 \mathrm{DM}$.

- SGLT 2 inhibitors with risks of dehydration, ketoacidosis, and fungal infections which are in any case not indicated in emergency situations should be stopped.

- The glitazones cause fluid retention and therefore may exacerbate the cytokine storm-induced lung pathology and should therefore be stopped!

- Metformin can cause lactic acidosis in the presence of acute infections!

- Since DPP inhibitors can also affect diverse enzymes other than DPP-IV only, their use during unknown infections is best avoided!

- Insulin is the glucose-lowering therapy of choice for acute hyperglycemia. Hyperglycemia is often exacerbated or precipitated by the steroid therapy used for the cytokine storm. With insulin preprogrammed pump therapy, only short acting insulins are used.

- Occasional de novo diabetic ketoacidosis has been observed in persons not known to be diabetic!

- Potent drugs, including sulfonylureas and long acting insulins which are known to cause hypoglycemia are also best avoided.

Abbreviations: DPP-IV, dipeptidyl peptidase-4; SGLT, sodium-dependent glucose cotransporters.

The jury is still out on the use of HCQ. Recent Mediscape articles just published ${ }^{35-39}$ exemplify the divergent opinions. Whether HCQ is beneficial for the COVID illness is debatable, but with suggested electrocardiogram monitoring it does not appear to be dangerous! In India both ICMR(Indian Council of Medical Research) and AIIMS do advocate their use, albeit with EKG monitoring and caution, and so do the present authors, based on their safe use in rheumatoid arthritis and in diabetes prior to COVID.

\section{Antivirals for COVID Therapy}

\section{Remdesivir}

A preliminary report by Beigel et $\mathrm{al}^{40}$ from National Institutes of Health based on 1,063 subjects suggested 30\% reduction in hospitalization time with intravenous (IV) remdesivir (200 mg day 1 loading dose, followed by $100 \mathrm{mg}$ IV daily for up to 9 additional days). However, further data are clearly needed especially with regard to cure rate/viral burden, etc.

\section{Lopinavir/Ritonavir}

A lopinavir/ritonavir combination used earlier in HIV (human immunodeficiency virus) patients is also being evaluated for COVID-19 patients. The results have been rather 
Box E Changing clinical pictures and multisystem features

Observing the changing clinical picture of the COVID-19 pandemic, from a flu-like lung illness to a multisystem picture, one is reminded of Lorraine Daston's historical perspective ${ }^{53}$

To quote her:

"Historically,

it is natural to cast about for answers at the dawn of a pandemic.

At moments of extreme scientific uncertainty,

observation, usually treated as the poor relation of experiment and statistics in science,

comes into its own.

Confronting a new disease, doctors have no choice but to turn to suggestive single cases, striking anomalies, partial patterns.

Slowly, as our ideas about what works and what does not help tell us

what to test, what to count, the picture clarifies.

Until then, we are back in the seventeenth century, the age of ground-zero empiricism, and observing as if our lives depended on it.

One patient at a time, we have to work our way into the present!"

Why the multisystem involvement?

- Wide distribution of ACE 2 receptors through which coronavirus enters cells!

- Secondary effects of hypoxia and a generalized acute inflammatory state and the toxic cytokine storm!

- Hypercoagulability, high C-reactive protein, ferritin, D-dimer, N/L ratio: reflecting lymphopenia + neutrophilia, fever.

Gastrointestinal symptoms were noted in the Wuhan early reports, but today some subjects have a purely gastrointestinal presentation, long before the chest symptoms.

Ageusia: loss of taste (for sweet and salty but sour taste is retained), often with loss of smell (anosmia), diarrhea, nausea/vomiting, abdominal pain, and anorexia can occur.

Anosmia was blamed for the direct involvement of the nasal mucosa by SARS-CoV-2. It was also attributed to direct brain invasion of the olfactory cortex from the nasopharynx. Does this occur through the cribriform plate in the skull base-may merit study!

Other neurological features that are being noticed include headache, ataxia, and even full-fledged strokes. Surprisingly, these are common in young subjects; abnormal zombie like behavior and confusion have been noted, and encephalopathy has been thought to be present. Renal involvement can occur, and usually carries a bad prognosis, despite dialysis! Acute kidney injury, albuminuria, and raised creatinine can occur.

Dermatological features of COVID-19

- Skin rash

- Chilblains

- Thickened skin on soles analogous to frostbite lesions

- Itching

- Red skin

Rash similar to that of frequently reported in Kawasaki syndrome in children: when present, high fatality rate has been observed.

Endocrine features:

- Diabetic ketoacidosis in a previously nondiabetic subject.

- TSH rise despite relatively normal T3 and T4.

- Subacute thyroiditis: single case just reported from Italy: woman aged 18 y who presented with fever, neck pain that radiated to the jaw, and palpitation occurring $15 \mathrm{~d}$ after testing positive for SARS-CoV-2 on oropharyngeal swab. ${ }^{29}$

- Increased heart rate and a painful and enlarged thyroid on palpation. FT4 and FT3 were high, TSH was undetectable, and inflammatory markers and white blood cell count were elevated. Bilateral and diffuse hypoechoic areas were detected at neck ultrasound.

One month earlier, thyroid function and imaging were both normal. "We diagnosed subacute thyroiditis and the patient started prednisone," the authors wrote: "Neck pain and fever recovered within $2 \mathrm{~d}$ and the remaining symptoms within 1 week. Thyroid function and inflammatory markers normalized in 40 days." ${ }^{29}$

Abbreviations: COVID-19, coronavirus disease 2019; SARS-CoV-2, severe acute respiratory syndrome coronavirus-2; TSH, thyroid stimulating hormone.

disappointing when tried in a difficult group by Cao et al from Wuhan. ${ }^{41}$ The NEJM commented thus:

"We have few specific tools to control the growing epidemic and treat those who are sick. We rely on quarantine, isolation, and infection-control measures to prevent disease spread and on supportive care for those who become ill. What we lack is a specific antiviral agent to treat the infected and, optimally, decrease viral shedding and subsequent transmission.

One antiviral-drug candidate is a combination of the HIV protease inhibitors lopinavir and ritonavir. Lopinavir, which acts against the viral 3CL protease, has modest antiviral activity against SARS-CoV-2. Together with ritonavir, which increases drug bioavailability, it is in clinical trials, along with the immunomodulator interferon $\beta-1 b$, for the treatment of Middle East respiratory syndrome (MERS). What makes lopinavir/ritonavir, particularly attractive is that it is widely available and manufacturable to scale and that it could be prescribed immediately. In fact, there are several case reports and case series where this agent is being used against COVID-19. But does it work?

This is the question that motivated Cao and colleagues to perform an urgent randomized clinical trial of the efficacy of lopinavir/ritonavir in patients with COVID-19 in Wuhan, China, the epicenter of the outbreak.... The investigators 
Box F Managing inflammatory coagulopathy and disseminated intravascular coagulation

Inflammatory hypercoagulopathy prominent in COVID-19:

- Low molecular weight heparin is widely used.

- Tissue plasminogen activator (TPA) reopens pulmonary vasculature, at least temporarily; hypoxia improves but relapses readily.

- Antithrombin-III (AT-III) advised in Japan: but evidence is weak. Also used there in sepsis and disseminated intravascular coagulopathy (DIC).

- Hypoxia improves but recurs after TPA withdrawal!

- Combination of heparin and AT-III therapy may cause bleeding episodes.

- It might be time for reconsidering the interaction and modulation of different connected systems, e.g., coagulation, fibrinolysis, Kallikrein-Kinin, complement and immunity (cytokine storm). An algorithm for optimizing therapy has been proposed by Seitz and Schram recently in 2020, who have described rational strategies for attenuating DIC in COVID-19. ${ }^{23}$

Abbreviation: COVID-19, coronavirus disease 2019.

Box G Acute phase reactants used for diagnosis of COVID-19

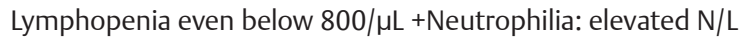
ratios

- Elevated Troponin-T

- Elevated D-dimer $>1,000 \mathrm{ng} / \mathrm{mL}$

- Elevated CPK-MB greater than two times normal

- Elevated C-reactive protein (CRP)

- Raised ferritin (>500 $\mu \mathrm{g})$

- Elevated LDH, ALT (SGPT), and AST (SGOT)

- Elevated IL-6

- Elevated Von Willebrand factor (VWF), consistent with endothelial injury

- Increased factor VIII activity

Abbreviations: ALT, alanine aminotransferase; AST, aspartate aminotransferase; CPK-MB, creatine kinase myocardial band; IL-6, interleukin-6; LDH, lactate dehydrogenase; SGOT, serum glutamic oxaloacetic transaminase; SGPT, serum glutamic pyruvic transaminase.

recruited patients who had an oxygen saturation of $94 \%$ or less while they were breathing ambient air or a ratio of the partial pressure of oxygen to the fraction of inspired oxygen of less than $300 \mathrm{~mm} \mathrm{Hg}$ and who were receiving a range of ventilatory support modes, from nothing to mechanical ventilation or extracorporeal membrane oxygenation (ECMO). .... All the patients received standard care, and half were randomly assigned to receive lopinavir/ritonavir for 14 days. The primary end point was the time to clinical improvement, defined as the time from randomization to either discharge from the hospital or improvement on a multifactorial set of prespecified criteria, whichever came first. The trial aimed to enroll 160 patients......

Unfortunately, the trial results were disappointing. No benefit was observed in the primary end point of time to clinical improvement: both groups required a median of
Box $\mathbf{H}$ Managing the cytokine storm in COVID-19

- When in doubt.

- Order C-reactive protein/troponin-T/C-reactive protein.

- Dexamethasone along with anticoagulation is the sheet anchor (details of dosage are outlined in the final paragraph of this review outlining current management as of August 20, 2020!).

- Use remdesivir/anti-IL-6 antibodies if available.

- Inotropes: noradrenaline preferred over dopamine.

- Hydroxychloroquine (HCQ)/azithromycin after checking QT intervals and watching for changes on the tele-EKG strip without recording formal EKG/Echo (ICMR guidelines as issued).

- Minimize patient handling.

- Convalescent serum and injectable hyaluronidase /niacin have been reported beneficial in anecdotal cases.

Abbreviations: EKG, electrocardiography; ICMR, Indian Council of Medical Research.

Box I Factors increasing morbidity in COVID-19

- Diabetes mellitus

- Hypertension

- Cancer

- Preexisting heart disease

- High BMI (even more so than merely diabetes!)

- Black, in contrast to Caucasian genetic background

- Male sex as compared with female sex

- Group A blood type

Abbreviations: BMI, body mass index; COVID-19, coronavirus disease 2019.

16 days. But the results for certain secondary end points are intriguing. A slightly lower number of deaths was seen in the lopinavir/ritonavir group, although this observation is difficult to interpret....both groups were heterogeneous and received various additional treatments, including other pharmacologic interventions such as interferon (11\%) and glucocorticoids (34\%)....

Tellingly, though, there was no discernible effect on viral shedding. Since the drug is supposed to act as a direct inhibitor of viral replication, the inability to suppress the viral load and the persistent detection of viral nucleic acid strongly suggest that it did not have the activity desired.... Two major factors may be in play. First, the authors chose a particularly challenging population. The patients recruited for the study were late in infection and already had considerable tissue damage (as evidenced by compromised lung function and $25 \%$ mortality in the control group). Even highly active antibacterial agents have limited efficacy in advanced bacterial pneumonia. Second, lopinavir simply is not particularly potent against SARS-CoV-2. The concentration necessary to inhibit viral replication is relatively high as compared with the serum levels found in patients treated with lopinavir/ritonavir....In addition, $42 \%$ of the patients were viral load-positive at day 28 , but the quantitative data at that point show that the levels were low, probably near 
Box J Antibody testing

- "Point-of-care and laboratory-based serologic assays for SARS-CoV-2 now have Emergency Use Authorization (EUA) by the U.S. Food and Drug Administration (FDA), and their performance has been independently reviewed.

- Currently, there is no identified advantage of assays whether they test for $\lg G$, IgM, and $\lg G$, or total antibody.

- It is important to minimize false positive test results by choosing an assay with high specificity and by testing populations and individuals with an elevated likelihood of previous exposure to SARS-CoV-2. Alternatively, an orthogonal testing algorithm (i.e., employing two independent tests in sequence when the first test yields a positive result) can be used when the expected positive predictive value of a single test is low.

- Antibodies most commonly become detectable 1-3 wk after symptom onset, at which time evidence suggests that infectiousness likely is greatly decreased and that some degree of immunity from future infection has developed. However, additional data are needed before modifying public health recommendations based on serologic test results, including decisions on discontinuing physical distancing and using personal protective equipment (PPE)."

\section{Antigenic targets}

The two major antigenic targets of SARS-CoV-2 virus against which antibodies are detected are spike glycoprotein (S) and nucleocapsid phosphoprotein $(\mathrm{N})$. While $\mathrm{S}$ protein is essential for virus entry and is present on the viral surface, $\mathrm{N}$ protein is the most abundantly expressed immunodominant protein that interacts with RNA. Multiple forms of S protein-full-length (S1+S2) or partial (S1 domain or receptor binding domain $[\mathrm{RBD}]$ )-are used as antigens. The protein target determines cross-reactivity and specificity because $\mathrm{N}$ is more conserved across coronaviruses than S, and within S, RBD is more conserved than S1 or full-length $\mathrm{S}$.

\section{Types of antibody testing}

Different types of antibody testing assays can be used to determine different aspects of immune response and functionality of antibodies. The tests can be broadly classified to detect either binding or neutralizing antibodies.

- Binding antibody detection: These tests use purified proteins of SARS-CoV-2, not live virus, and can be performed in lower biosafety level laboratories (e.g., BSL-2). With specific reagents, individual antibody types, like $\lg G$, IgM, and IgA, can be determined. In general, IgM is one of the first types of antibodies produced after infection and is most useful for determining recent infection, while IgG generally develops after IgM and may remain detectable for months or years. IgA is important for mucosal immunity and can be detected in mucous secretions like saliva in addition to blood, though its significance in this disease is still to be determined. Depending on the complexity of assays, these tests can be performed rapidly (less than 30 min) in a field setting or in a few hours in a laboratory.

Tests that detect binding antibodies fall into two broad categories:

1. Point-of-care (POC) tests generally are lateral flow devices that detect IgG or lgG and IgM, or total antibody in serum, plasma, whole blood, and/or saliva. An advantage of some point-of-care tests using whole blood is that they can be performed on blood samples obtained by fingerstick rather than venipuncture.

2. Laboratory tests use ELISA (enzyme-linked immunosorbent assay) or CIA (chemiluminescent immunoassay) methods for antibody detection, which for some assays may require trained laboratory technicians and specialized instruments. Based on the reagents, IgG, IgM, and IgA can be detected separately or combined as total antibody titer.

- $\quad$ Neutralizing antibody detection: FDA has not yet authorized the use of neutralization tests (NT) for SARS-CoV-2. NT determine the functional ability of antibodies to prevent infection of virus in vitro. The test involves incubating serum or plasma with live virus followed by infection and incubation of cells. Testing will require either BSL-3 or BSL-2 laboratories, depending on what form of the SARS-CoV-2 virus is used.

Two types of neutralization tests are conducted.

1. Virus neutralization tests (VNT), such as the plaque-reduction neutralization test (PRNT) and microneutralization, use a SARSCoV-2 virus from a clinical isolate or recombinant SARS-CoV-2 expressing reporter proteins. This testing requires BSL-3 laboratories and may take up to $5 \mathrm{~d}$ to complete.

2. Pseudovirus neutralization tests ( $\mathrm{pVNT}$ ) use recombinant pseudoviruses (like vesicular stomatitis virus, VSV) that incorporate the S protein of SARS-CoV-2. This testing can be performed in BSL-2 laboratories depending on the VSV strain used.

Abbreviations: IgA, immunoglobulin A; IgG, immunoglobulin G; IgM, immunoglobulin C; RNA, ribonucleic acid; SARS-CoV-2, severe acute respiratory syndrome coronavirus- 2 .

the threshold of detection. Since the test detects nucleic acid, positive results do not necessarily indicate the production of infectious virus. These data suggest that assessing transmissibility after recovery from severe disease will be a priority to help control transmission..."

\section{Favipiravir, Ostelamivir/EIDD 2801}

Other antivirals, such as favipiravir, are also being studied... This is an Indian generic version of the Japanese drug Avigan which was recently approved in Russia. This drug and DAS 181 are being evaluated.

Oseltamivir (Tamiflu) has not been reported to be effective, though it was very useful in earlier swine flu epidemics!

EIDD 2801: This is another promising antiviral from the Emory University, resembling remdesivir, which has just received FDA (Food and Drug Administration) approval for human clinical trials (EMORY news center April 7, 2020). ${ }^{42}$ Unlike remdesivir, which has to be given 
Box K Prehospital management of suspected COVID-19 patient and COVID-19 patient contacts

- Do not panic

- Watch $\mathrm{pO}_{2}$ with pulse oximeter

- Test for acute phase reactants: ferritin, troponin-T, C-reactive protein, neutrophil/lymphocyte ratio, measure body temperature daily preferably with an infrared thermometer

- Quarantine/self-isolate /including PPE-masks/plastic shields/ gloves, and gowns.

- Stay in touch with your doctor (telemedicine).

- Play solitaire

- Pray

- Read

- Listen to music/see TV

- Repeat COVID-19 testing at 7/14/28 days

Abbreviations: COVID-19, coronavirus disease 2019; PPE, personal protective equipment.

intravenously, EIDD-2801 can be given orally as a pill. It is an orally bioavailable prodrug of the synthetic ribonucleoside derivative of $\beta$-D-N4 hydroxy cytidine (NHC/EIDD-1931) as 5-isopopylester (EIDD2801). Its parent compound NHC/ EIDD-1931 has been shown to have broad spectrum antiviral activity against SARS-CoV-2, MERS-CoV, and SARS-CoV. If EIDD-2801 is shown to be safe and effective, people could take it at home rather than in a hospital. That would allow EIDD-2801 to be taken earlier in the course of the disease, killing off the virus before it wreaks havoc on the body.

EIDD-2801's other intriguing feature is that it appears to have a high barrier to resistance. Drugs can force viruses to quickly develop mutants that are not affected by the drug, which then makes the drug obsolete. But EIDD-2801 has not prompted that sort of resistance in laboratory tests despite efforts to coerce such mutants to arise. With hints that EIDD-2801 and its active metabolite, the triphosphate of EIDD-1931, might be able to fight other RNA viruses, Painter and his collaborators began testing it against Ebola, chikungunya, and influenza. It knocked them all out. For example, just three doses of EIDD-2801 could save ferrets (animals commonly used to study human respiratory viruses) infected with influenza from the 2009 H1N1 pandemic.

\section{Interferon-ALPHA-2b}

Recently interferon- $\alpha-2 b$ is attracting attention in viral treatment. ${ }^{43}$

\section{Corticosteroids}

Logically corticosteroids were suggested to reduce the inflammation of the cytokine storm. After the Recovery trials from Oxford showed reduction in mortality they are now in widespread use! The penultimate paragraph on COVID management in August 2020 describes current usage protocols.

\section{Agents That Are Promising}

Colostrum of bovine origin, various folk medicines both from Chinese and Ayurvedic medicine, (including Giloy and Tulsi-Tinospora cordifolia and Ocimum sanctum) as well as Homeopathy are undergoing trials as preventive agents.

Ivermectin (12 mg weekly), high doses of vitamin C, zinc, selenium, inositol, and niacin all have been suggested as preventives or as therapy. Unlike HCQ there are no toxic effects reported. After robust in vitro efficacy reports from Monash University, Australia, ${ }^{44}$ Alam ${ }^{45}$ from Bangladesh claimed that 60 patients treated with a combination of ivermectin and doxycycline recovered within 4 days. The data were being prepared for publication.

Unlike azithromycin, doxycycline has not been reported to cause QTc prolongation.

We suggest that it would appear logical to try out low cost combinations of doxycycline, ivermectin, HCQ, EIDD 2801, and azithromycin-all being oral drugs, not needing costly, hazardous hospitalization.

Results of Indian studies with herbal and homeopathic drugs (Gupta, Pradip: Personal Communication) are awaited. It is hoped that these are conducted with scientific rigor.

\section{Convalescent Serum}

- This has been suggested as a safe intervention in sick patients. Casdevali et $\mathrm{al}^{46}$ give an excellent overview of discussed risks and benefits. Measurement of antibody levels and nature of antibody present would be useful, but empirically this is being used with benefit at various centers in India, and ICMR in India and the FDA in United States have approved this as compassionate use. No clinical trials are feasible in such extremely sick patients!

\section{IL-6 Inhibition}

- Measurement of interleukin-6 (IL-6) as an acute phase index of a cytokine storm often shows very high levels. tumor necrosis factor- $\alpha$ and IL 1 are not as high! Tocilizumab is a monoclonal antibody that blocks both membrane-bound and circulating IL-6 receptor. ${ }^{47}$ It is being tried at some centers to treat the cytokine storm. Sarilumab is another anti-IL-6 monoclonal being evaluated.

\section{Other Agents Being Evaluated ${ }^{38}$}

Vitamin D to enhance macrophage function, IL-1 inhibitors such as anakinra, nitric oxide, JAK inhibitors, baricitinib, pacritinib, ruxolitinib NMDA (N-methyl-D-aspartate), mesenchymal cells are other agents undergoing phase I/II or III trials but they are still far from being in routine use! Dipyridamole, monoclonal antibodies, famotidine, thymosin alpha, acalabrutinib, BCG (Bacillus Calmette-Guérin) vaccination, p38MAPK inhibitors, and colchicine have also been referred to in recent reviews. ${ }^{39,48}$ 
Postscript: Outline of Present-Day COVID Management as of August 20, 2020

This is partly based on a recent review by David Cenmino in Medscape updated August 20, 2020! COVID-19 treatment and management ${ }^{49}$ as well as a Karnataka Medical Association Guidance note dated August 14, 2020.50

Once COVID is diagnosed or strongly suspected, obtain a baseline pulse oximetric pO2, $\mathrm{Hb}$, total leukocyte count, differential leukocyte count (to estimate neutrophil/lymphocyte ratio), erythrocyte sedimentation rate (ESR), C-reactive protein, D-dimer, IL6 (if available), lactate dehydrogenase (LDH), ferritin, Trop T, baseline liver, and kidney function tests especially SGPT or serum glutamic pyruvic transaminase (ALT or alanine aminotransferase), S creatinine, ECG, baseline chest $\mathrm{X}$-ray, and CT chest.

Even CT changes may be late but CRP and LDH are the first to rise. Clinically the three dangerous symptoms are fever, myalgia, and exhaustion which indicate high inflammation in the body. Feverish feeling without actual fever may occur!

Nasopharyngeal smear may be negative, even if supplemented by RT-PCR and also antibody tests. Best time for RT-PCR after symptom onset is fifth to eighth day. False negatives are least during this period. RT-PCR may be negative in late stages of infection. One must therefore base one's diagnosis on clinical, epidemiological, and radiological clues. The $r$ replication score in RT-PCR test indicates how infective a subject is for others. Any score above 25 is generally noninfectious especially if it is beyond 10 days and there have been no symptoms for 3 consecutive days. Viral replication generally stops by nineth day which implies that if one expects any benefit of antiviral therapy it should be given within 9 days. There is doubt regarding the efficacy of remdesivir, if given late.

It is the inflammation which damages not the virus. COVID, systemic lupus erythematosus, antiphospholipd syndrome, type 1 diabetes mellitus macrophage activation syndrome, and hemophagocytic lymphohistiocytosis are all similar... Host immune system reacts against its own tissues... ... Frustrated phagocytosis “. occurs when the antigen cannot be eliminated leading to hyperactivation of macrophages and lymphocytes leading to destruction of tissues.

Steroids and anticoagulation are the corner stones of treatment. At present steroids are recommended for people who become hypoxic. But one should start steroids early irrespective of hypoxia if CRP and other inflammatory markers are elevated.

Oral prednisolone $20 \mathrm{mg}$ or dexamethasone $4 \mathrm{mg} / \mathrm{d}$ for 5 to 7 days is used if the symptoms are mild. Steroids not only make the patient feel better but more importantly prevent long-term lung fibrosis, and its specter of lung transplantation! In severe cases, injection of methylprednisolone 40-mg intravenous bd or tid is based on weight and severity of hypoxia. Steroids do cause hyperglycemia and may precipitate diabetes but this is easily managed with soluble insulin. One changes to oral dexa once the course of antiviral is finished. Steroids may be needed for 2 to 3 weeks if hypoxia is present. Since steroids are diabetogenic, sugars must be monitored regularly as long as patients are on steroids.
With regard to anticoagulation, enoxaparin (Inj Clexane) used is $0.5 \mathrm{mg} \mathrm{s} / \mathrm{c} \mathrm{bd}$ if D-dimer is normal, and $1 \mathrm{mg} \mathrm{s} / \mathrm{c} \mathrm{bd}$ if D-dimer is elevated. After 5 days, one can switch to oral anticoagulant Tab rivaroxaban $10 \mathrm{mg}$ od for 4 weeks. It is debated when to start anticoagulants. Better to start it early if CT changes are seen, as the changes in CT are actually microvascular thrombi. If oxygen is needed, anticoagulation must be continued for 6 weeks. Early steroids and anticoagulant will prevent long-term complications, even if they do not show benefit in the acute phase! Both are mandatory if there is hypoxia.

It has been opined that HCQ should not be combined with remdesivir.

It is mandatory to monitor CRP, D-Dimer every alternate day till patient is in hospital: IL6 on day 5 and day 8. Any rise in IL6 is a marker for an impending cytokine storm and it might be useful to start tocilizumab if symptoms worsen. IL6 can return to normal with steroids and anticoagulant. If tocilizumab is given procalcitonin should be monitored for secondary infection and the risk of infection is present for the next 2 weeks. Once tocilizumab is given patients may not manifest fever or raise counts despite existence of infection. Antibiotics may be used depending on the community usage. Doxycycline is safe. Azithromycin can cause QTc prolongation and should not be used in combination with hydroxychloroquine.

With regard to prognosis, the dangerous period is 8 to 12 days, when mortality is the greatest. Rarely late cytokine storms after 12 days are seen.

But if treatment is started early with steroids and anticoagulants complications are unlikely. Again, to reiterate it is the inflammation which kills, not the virus. If one hits inflammation and coagulopathy hard and early, one should, Deo Gratia, avoid complications, and the body will eliminate the virus.

Proning makes a marked difference in managing hypoxia.

Radiologically, CT chest need not be repeated after the base line because excessive radiation may exacerbate lung injury: repeat X-ray chest after 3 days suffices, and artificial intelligence is being looked at for interpreting these X-rays. Remember radiological changes may take long to clear, as these shadows are lung clots not inflammation!

Improvement in oxygen levels is the marker for clinical improvement. and the most reliable indicator of patient status.

If antibody levels are good, the subject's convalescent serum may help a critical ill patient of the same blood group recover!

Antibody Status and Return to Work

About 10 to $15 \%$ of the patients do not develop antibodies post COVID. Perhaps some subjects do not develop antibodies, or develop some T-cell immunity, or have dominant IgA antibody response in respiratory mucosa instead of a t systemic IgG response

Relapse can occur: therefore, continue to follow anti COVID precautions! Follow strict safety protocols post COVID too.

\section{Summary}

The battle with the coronavirus has just been joined. Let us pray that men of medicine and science continue to improve management. The struggle to obtain a safe vaccine is good 
but let us remember that defeating the earlier optimism in some diseases such as HIV and Dengue, we still do not have a vaccine. For rabies, smallpox, and tuberculosis (TB) on the other hand vaccines dating back to Pasteur and Jennings have been spectacularly successful. BCG inoculation modified the TB onslaught and reduced the death rates from tubercular meningitis, even though they did not eliminate TB. Also, the Holy Grail vaccines should not precipitate dangerous or lethal immune reactions!

Men of economics and industry and those leading the Government, should try to prepare for and prevent future pandemics, perhaps looking to what we have sketched out in the - Table 1. Recent CDC guidelines ${ }^{51}$ make instructive reading, while the nation gears up for the post lockdown era and the problems related to our hardworking migrant labor.

Social distancing and hand sanitation are interim measures, while cure/vaccine options are developed. All human beings including the Readers and Health Care Workers are requested to not only protect themselves but also set examples!

\section{Conflict of Interest}

None declared.

\section{References}

1 Chidinma Chima Melton. Management of Critically Ill COVID-19 Patients. What Are the Protocols at UCLA Health? Vu Medi April 2020, 28-36; 2020

2 Cordon-Cardo C. COVID-19: What Is the Benefit of Antithrombotic Therapies \& What Is the Prothrombotic Nature of the Disease? Does Hypoxia Induce Organ Damage or Is It a Multi-organ Disease? New York, NY: Mount Sinai Vu MediVideo; 2020; 2020

3 Cordon-Cardo C, Garcia-Sastre A. COVID-19: What Determines a Person's Immune Response? When Is the Right Time to Do Antibody Testing \& How Do You Determine What Degree of Antibody Response Is Protective? New York, NY: Mount Sinai Vu Medi Video; 2020

4 Cordon-Cardo C, Patel G. COVID-19 Medication Responses from Mount Sinai: What Should We Know about Hydroxychloroquine, Steroids, Remdesivir and Plasma? In What Situations Is Plasma Treatment Effective? Do We Know What Doses to Give? New York, NY: Mount Sinai Vu Medi Video; 2020

5 Cordon-Cardo C, et al. COVID-19: Will There Be a Second Wave of Infection and Will the Virus Virulence Change in Time? New York, NY: Mount Sinai Vu MediVideo, May 2020; 2020

6 Cordon-Cardo C, et al. COVID-19 Testing Challenges: What Is the Importance of Testing for Diagnosis and Stratifying Patients by Viral Load? What Is the Possibility of a False Positive or False Negative Test? New York, NY: Mount Sinai Vu MediVideo, May 2020; 2020

7 Garcia Sastre A, Narula J. COVID-19: What Are Most of the Vaccines Based On\& What is the Main Focus in Neutralizing Antibodies Against the Receptor Binding Protein? New York, NY: Mount Sinai Vu MediVideo, May 2020; 2020

8 Griffin D. COVID-19 Patients: Which Drug to Prescribe, When, and to Whom? What Are Some Cardiac, Cognitive \& Neurological Clinical Manifestations, and Who Should Receive Anticoagulants. Vu MediVideo, April 2020; 2020
9 Jaffe A. What Does Elevated Troponin Mean in COVID-19? Vu MediVideo, May 2020; 2020

10 Jain T, et al. Potential Effects of COVID-19 on the Cardiovascular System: How to Treat ST-Elevation on EKG? Why Are STEMIs Going Down During the Pandemic? New York, NY: Mount Sinai Vu MediVideo, May 2020; 2020

11 Mahmood K, et al. ARDS and COVID-19 in an LVAD Patient: Should We Look at Percent Change in LDH, Ferritin and CRP Values in A Cytokine Storm to Assess Severity? What Cardiac Manifestations Do We Need to Keep an Eye on? New York, NY: Mount Sinai Vu MediVideo, May 2020; 2020

12 Mascarenhas J. Can Pacritinib Be Used to Treat Cytokine Storm in Patients With COVID-19? What Was the Primary End Point of the Trial \& Which Patients Are Eligible for the Treatment? New York, NY: Mount Sinai Vu MediVideo, May 2020; 2020

13 Moll S. COVID-19 Coagulopathy: What is the PE and DVT Risk? How Should We Optimize the Anticoagulation Therapy Algorithm?(Describes Current UNC Algorithm) Vu MediVideo, May 2020; 2020

14 Narula J, Sharma S. STEMI Patients with COVID-19: What Cardiovascular Manifestation Are Expected in Patients? What Is the Management Protocol to Treat Them? New York, NY: Mount Sinai Vu MediVideo, May 2020; 2020

15 bok15. Olin J. COVID-19: What Types of Thrombosis Are Commonly Seen? Are Anticoagulants Effective \& How to Use them? New York, NY: Mount Sinai Vu MediVideo, May 2020; 2020 /bok

16 Olin J, et al. COVID-19 and Stroke: Is Large-Vessel Stroke a Presenting Feature of COVID-19: in the Young? Should We Monitor These Patients with Blood Work, Antiplatelet, or Antithrombotic Therapy? Is There a High Bleeding Complication Rate? New York, NY: Mount Sinai Vu MediVideo, May 2020; 2020

17 Olin J, et al. What Biomarkers Are Recommend for Looking for Pulmonary Embolism in COVID-19 Infected Patients? Are They Good for Diagnostic Purposes? New York, NY: Mount Sinai Vu MediVideo, May 2020; 2020

18 Olin J, et al. COVID-19: Are You Requiring PCR Testing for All In-person Encounters and What Is the Turnaround Time? How Do You Protect Staff? New York, NY: Mount Sinai Vu MediVideo, May 2020; 2020

19 Olin J, et al. Venous Thromboembolism \& COVID-19: Has There Been an Increased Incidence at Mount Sinai Health System? What About Performing Mechanical Thrombectomies in Critically Ill Patients? New York, NY: Mount Sinai Vu MediVideo, May 06, 2020:53; 2020

20 Olin J, et al. What Is the Role of Endothelial Dysfunction in the Vascular Complications Associated with COVID-19 Patients? Why Do We See So Much Thrombosis in These Patients? New York, NY: Mount Sinai Vu MediVideo, May 2020; 2020

21 Osterholm M, hVuMedi, May 2020: Possible Scenarios for the Course of the COVID-19 Pandemic: Are We Prepared for at Least Another 18 to 24 Months of Significant COVID-19 Activity? VuMediVideo, May 2020; 2020

22 Reddy V, Bokhari M. Electrophysiological Analysis of Two Cases of COVID-19 Patients: How do Plaquenil, Chloroquine and Azithromycin Effect the Cardiovascular System? What Causes Troponin Elevation in COVID-19 Patients with Myocardial Injury? New York, NY: Mount Sinai Vu MediVideo, May 2020; 2020

23 Seitz and Schram. Rationale for Developing Strategies for Attenuating DIC in COVID-19. Vu MediVideo, May 2020; 2020

24 Serrao GL2020: Refractory ARDS \& COVID-19: Why Should VV ECMO Work and When Should It Be Used? Which Patients Are Good Candidates for ECMO? New York, NY: Mount Sinai Vu MediVideo, May 2020; 2020 
25 Serrao G. ECMO for COVID-19: Should It Be Used to Treat ARDS \& How to Deal with Recirculation Problems? Why Is It Hard to Use VV ECMO in Patients with High Cardiac Output States? Has the Use of Remdesivir in Combination with ECMO Shown to Be Successful? New York, NY: Mount Sinai Vu MediVideo, May 2020; 2020

26 Serrao G. Shock in COVID-19 Era: How to Treat Different Types of Circulatory Shock? Does Placing Univentricular Support Risk Precipitate Frank Failure of the Other Ventricle? Why is IABP the Preferred Approach over ECMO? New York, NY: Mount Sinai Vu MediVideo, May 2020; 2020

27 Warner JP. (Codman Shoulder Society):Post-Pandemic Orthopedic Care: Challenges \&Opportunities. VuMediVideo, May 2020; 2020

28 Herold T, Jurinovic V, Arnreich C, et al. Elevated levels of IL-6 and CRP predict the need for mechanical ventilation in COVID-19. J Allergy Clin Immunol 2020;146(1):128-136.e4

29 Brancatella A, Ricci D, Viola N, Sgrò D, Santini F, Latrofa F. Subacute thyroiditis after Sars-COV-2 infection. J Clin Endocrinol Metab 2020;105(7):2367-2370

30 Hick JL, Biddinger PD. Novel coronavirus and old lessonspreparing the health system for the pandemic. N Engl J Med 2020;382(20):e55

31 Islam MT, Fleischer JW. Distinguishing L and $\mathrm{H}$ phenotypes of COVID-19 using a single x-ray image (Prepublication). Available at: https://doi.org/10.1101/2020.04.27.20081984. Accessed May 3, 2020

32 Interim Guidance for Rapid Antigen Testing for SARS-CoV-2. Available at: https://www.cdc.gov/coronavirus/2019-ncov/ lab/resources/antibody-tests-guidelines.html and July 27 2020 update https://www.cdc.gov/coronavirus/2019-ncov/ hcp/testing-overview.html and https://www.cdc.gov/coronavirus/2019-ncov/lab/resources/antigen-tests-guidelines.html. Accessed August 16, 2020

33 Director General WHO. Announcing suspension of hydroxychloroquine arm of SOLIDARITY trial; 2020. Available at: https://www.who.int/dg/speeches/detail/WHO-DirectorGenerals'-opening-remarks-at-the-media-briefing-onCOVID-19-25-May 2020

34 Hazra DK. Letter to Prof Shashank Joshi, Dean, Indian College of Physicians, April 7, 2020; 2020

35 Risch HA. The key to defeating COVID 19 already exists: We need to start using it. Newsweek August 22, 2020; 2020

36 Risch HA. Early treatment of symptomatic high risk COVID 19 patients that should be ramped up immediately as key to the pandemic control. Am J Epidemiol 2020;27

37 Perry Wilson F. HCQ RCTs: ethically the choice is clear Medscape. Intern Med 2020;5

38 Bergman S, Treatment of Coronavirus COVID 19: Investigational Drugs and Other Therapies, updated Aug 5, 2020, Medscape; 2020
39 American College of Physicians. COVID-19: An ACP Physician's Guide + Resources. Available at: https://www.acponline.org/ clinical-information/clinical-resources-products/coronavirusdisease-2019-covid-19-information-for-internists

40 Beigel JH, Tomashek KM, Dodd LE, et al; ACTT-1 Study Group Members. Remdesivir for the treatment of COVID-19-preliminary report. N Engl J Med 2020;383(10):994

41 Cao B, Wang Y, Wen D, et al. A trial of lopinavir-ritonavir in adults hospitalized with severe COVID-19. N Engl J Med 2020;382(19):1787-1799

42 Emory News Center. FDA: Human Trials can Begin for Emory COVID-19 Antiviral. Available at: News emory.edu/ stories/2020/04/COVID EIDD 2801-FDA/index/html; 2020

43 Zhou Q, Chen V, Shannon CP, et al. Interferon- $\alpha 2 b$ treatment for COVID-19. Front Immunol 2020;11:1061

44 Caly L, Druce JD, Catton MG, Jans DA, Wagstaff KM. The FDA-approved drug ivermectin inhibits the replication of SARS-CoV-2 in vitro. Antiviral Res 2020;178:104787

45 Alam MT. Bangladesh Medical Team Says Ivermectin with Antibiotic Doxycycline Works to Treat COVID 19 Patients. New Delhi: Press Trust of India release May 19, 2020; 2020

46 Casadevali A, Joyner MJ, Pirofski LA. A randomized trial of convalescent plasma for COVID-19-potentially hopeful signs. JAMA 2020;324(5):455-457

47 Sebba A. Tocilizumab: the first interleukin-6-receptor inhibitor. Am J Health Syst Pharm 2008;65(15):1413-1418

48 American College of Physicians. Coronavirus Disease 2019 (COVID-19): Information for Internists. Available at: https:// www.acponline.org/clinical-information/clinical-resources-products/coronavirus-disease-2019-COVID-19-information-for-internists

49 Cenmino D. Corona Virus Disease 2019 (COVID-19) Treatment and management: Updated 20 August 2020. Medscape; 2020

50 Karnataka Medical Association Advice. August 14,2020. Available at: https://www.facebook.com/permalink.php?story_fbid=2510 $971889123445 \& \mathrm{id}=1383946881825957$

51 CDC Guidelines 2020: Guidance re housing and high risk workers; 2020. Available at: https://www.cdc. gov/coronavirus/2019-ncov/daily-life-coping/living-in-close-quarters and .html / https://www. cdc.gov/coronavirus/2019-ncov/community/high-risk-workers.html

52 Hazra DK And Katoch V M. Department of Science and Technology, Govt of India: Project Submission on National Therapeutic Biomolecule Centre; 2005

53 Daston L. Wikipedia and Max Planck Institute for the History of Science 2017: the History of Science and the History of Knowledge 2017;1(1):131-154; Accessed May 8, 2020 